\title{
Vaginal Undifferentiated Carcinoma
}

National Cancer Institute

\section{Source}

National Cancer Institute. Vaginal Undifferentiated Carcinoma. NCI Thesaurus. Code C40264.

A carcinoma that arises from the vagina and is characterized by the lack of specific cellular differentiation. 